\title{
Willingness-to-pay for services provided by the Clinical Services Improvement Project (CSI) in Egypt
}

John H. Bratt

Nahla G. Abdel-Tawab

Population Council

Magdi A. Ibrahim

Mohammed Edress

Follow this and additional works at: https://knowledgecommons.popcouncil.org/departments_sbsr-rh

Part of the Demography, Population, and Ecology Commons, International Public Health Commons, and the Women's Health Commons How does access to this work benefit you? Let us know!

\section{Recommended Citation}

Bratt, John H., Nahla G. Abdel-Tawab, Magdi A. Ibrahim, and Mohammed Edress. 2005. "Willingness-topay for services provided by the Clinical Services Improvement Project (CSI) in Egypt," FRONTIERS Final Report. Cairo: Population Council. 


\title{
Willingness-to-Pay for Services Provided by the Clinical Services Improvement Project (CSI) in Egypt
}

\author{
John H. Bratt, Family Health International \\ Nahla Abdel-Tawab, Population Council \\ Magdi A. Ibrahim, Cairo Demographic Center \\ Mohammed Edrees, Clinical Services Improvement \\ Project (CPI)
}

February 2005

This study was funded by the U.S. AGENCY FOR INTERNATIONAL DEVELOPMENT (USAID) under the terms of Cooperative Agreement Number HRN-A-00-98-00012-00 and Subagreement number AI03.63A. The opinions expressed herein are those of the authors and do not necessarily reflect the views of USAID. 


\section{Acknowledgement}

This report is the result of close collaboration between the FRONTIERS Program, the Clinical Services Improvement Project and the Cairo Demographic Center, with financial support from the USAID Mission in Cairo. The authors wish to thank Dr. Yehia ElHadidi, Population and Family Planning Undersecretary, for his support of the study and for his interest in the implications of its findings for the Egyptian Family Planning Program. The valuable input provided by Ms. Brenda Doe and Ms. Shadia Atteya of the USAID Mission in Cairo is deeply appreciated. The cooperation and enthusiasm of Mr. Mohamed Ibrahim, Dr. Sally Saher and the staff at CSI clinics are gratefully acknowledged. Special thanks go to Dr. Siham El-Sherif, Ms. Mona Tawfik and the interviewers at CDC for their remarkable efforts in data collection and analysis. The input received from colleagues at the FRONTIERS Program and Dr. Hussein Abdel-Aziz of the POLICY Project throughout the study are deeply appreciated. We would especially like to acknowledge Dr. Jim Foreit for his assistance in suggesting appropriate statistical tests. Last but not least, this study would not have been possible without the patience and candidness of the CSI clients who participated in the study. 


\section{Executive Summary}

Willingness to Pay (WTP) surveys are increasingly being used in reproductive health programs to predict the impact of price changes on revenues, utilization and client profile. The Clinical Services Improvement (CSI) project wanted to use a WTP survey to measure client reaction to price increases for selected services. CSI also was interested in knowing whether the actual behavior of clients matched their predicted behavior in the WTP survey. The FRONTIERS program worked with CSI and the Cairo Demographic Center (CDC) to carry out a WTP survey in 6 CSI clinics. Following the survey, CSI increased the price of DMPA injections and then tracked DMPA clients over a fourmonth period to determine which clients returned for subsequent injections and which did not.

Most clients said they would be willing to pay higher prices for CSI services, and WTP did not vary much by client economic status. If CSI were to implement the lower of the three price increases in the survey, total clinic revenues would increase, and a small number of CSI clients would seek services elsewhere. Client intention to discontinue family planning use because of higher prices was almost non-existent. Among DMPA clients, the level of agreement between stated and actual WTP was moderate; when noneconomic reasons for discontinuation were taken into account, however, agreement between client stated and actual WTP was much higher, both for individual clients and in the aggregate. WTP surveys have the potential to be useful tools for predicting client response to price increases, but predictions for methods like DMPA must be adjusted to account for non-economic reasons for discontinuation. 


\section{Introduction}

Concern about long-term sustainability of reproductive health programs in developing countries is prompting program managers to seek ways to control costs and generate additional income. Charging fees to clients is one element of an overall sustainability strategy. ${ }^{1}$ Economic theory, however, states that demand for a good or service usually declines when the price is increased; thus, there are concerns that recovering more costs from clients will bring programs into conflict with goals to increase utilization and coverage, and can undermine efforts to provide services to low-income clients. This dilemma between pursuit of program sustainability on the one hand and social mission on the other, combined with the relative inexperience of many program managers in setting fees, creates a sense of uncertainty and risk surrounding pricing decisions.

Decisions regarding price increases would be easier to make if program managers had the ability to predict likely impacts of price changes on utilization, revenues and composition of client population. Over the past three decades, economists have developed the contingent valuation (CV) method, a survey-based technique that asks respondents how much they would be willing to pay for a hypothetical change in the provision of a good or service (Mitchell and Carson, 1989). CV surveys have been widely used in environmental programs to quantify program benefits for cost-benefit analysis (CBA) and in some cases, to guide the process of setting fees for services (Hanemann, 1994); the technique has also been applied in recent years to healthcare (Diener, et al., 1998). WTP surveys employing the CV technique have already been used in several countries to predict demand for clinical services and social marketing products (Foreit and Foreit, 2003).

Managers of the Clinical Services Improvement (CSI) project - a private non-profit agency providing health services throughout Egypt - wanted to know whether their clients would be willing to pay higher prices for family planning and other reproductive health services. Previous studies found evidence of willingness to pay (WTP) in Egypt among clients of public-sector facilities. A study conducted by the Cairo Demographic Center (1997) showed that 60 percent of clients in Ministry of Health and Population (MOHP) clinics were willing to pay double the price they were then paying for family planning injectables. Also, the Interim Demographic and Health Survey (El-Zanaty \& Way, 2004) showed that about three-quarters of respondents (who are mostly MOHP clients) were willing to pay LE 5 for injectable contraceptives (LE 6.2 equivalent to US\$1 in 2004). CSI managers wished to measure client WTP for several specific price increases, and to know whether the actual purchasing behavior of individual clients

\footnotetext{
${ }^{1}$ In Egypt, cost recovery is particularly relevant to the national family planning program; demand for contraception is rising due to the increasing number of married women of reproductive age (MWRA), which is expected to reach 11 million in 2017. Also, USAID - the principal donor of family planning methods in Egypt for the last two decades - is gradually phasing out support for contraceptive commodities so that by 2007 all contraceptive methods will be purchased by the Government of Egypt (GOE). In order to ensure availability of contraceptive methods, the GOE may need to consider increasing prices of contraceptives.
} 
matched their stated WTP. ${ }^{2}$ If such predictive validity could be shown, CSI could be confident in using WTP surveys as a tool to make informed decisions about pricing of services and products.

\section{Objectives}

1. To measure stated WTP of clients of selected RH services provided by CSI;

2. To use information on client WTP to predict changes in clinic revenues and utilization;

3. To evaluate the predictive validity of a CV survey at the level of the individual client, among users of the injectable DMPA.

\section{Methods}

\section{Design Overview}

For the first objective, a WTP survey was administered to CSI clients visiting outpatient clinics to receive selected reproductive health services. For the second objective, survey results were used to simulate changes in revenues and utilization assuming various price changes. For the third objective, we used a panel design in which observed client behavior was used to validate client-stated intentions regarding an actual increase in the price of DMPA. DMPA clients were used for the validation study because their need for re-supply at a three-month interval ensures that a decision to return to the clinic occurs within a predictable timeframe. DMPA clients that failed to return after the price increase were interviewed to understand the role that the price increase played in their decision-making. All fieldwork activities for the study were conducted by the Cairo Demographic Center (CDC).

\section{Selection of Study Sites}

The study was conducted in six clinics of CSI, an affiliate of the Egyptian Family Planning Association. CSI provides services at prices that are substantially lower than those charged in the commercial sector. CSI clients are mostly lower middle class women who cannot afford to pay high commercial sector fees and who do not seek services from MOHP facilities. CSI is currently recovering 50 percent of its costs and is

\footnotetext{
${ }^{2}$ Predicted willingness to pay (WTP) is useful for decision-making only if it corresponds closely to subsequent client behavior. Few studies in developing countries have examined this issue. A 1995 study of WTP for connections to new water systems in India found good agreement between the predicted and observed proportions of households that connected to the system, but a shortcoming of this study was its small sample size. Onwujekwe (2004) compared predictive validity of three different question formats to measure WTP for insecticide-treated bednets in Nigeria. Although the author concludes that "the findings give confidence that the $\mathrm{CV}$ method is a valid and justifiable tool for determining peoples' valuations of goods and services" (p. 534), he also cautions against generalizing these results to other products and regions of Nigeria. Bratt et al (2004) conducted a validation study among injectables clients in El Salvador, and found low predictive validity at the level of the individual client; in the aggregate, however, predictive validity was high because individual prediction errors in one direction were cancelled out by prediction errors in the other direction.
} 
striving for full cost recovery by 2007 , when USAID completely phases out of the project.

Criteria for clinic selection included high volumes of DMPA clients (at least 60 clients per month), and at least three other high-volume RH services. ${ }^{3}$ Following is a listing of the study clinics and the corresponding governorates where the clinics are located.

\begin{tabular}{|l|l|}
\hline Clinic & Governorate \\
\hline Al-Ayat & Giza \\
\hline Benha & Qualyubia \\
\hline El-Delengat & Beheira \\
\hline Hosh Issa & Beheira \\
\hline Zagazig & Sharkeya \\
\hline Zefta & Gharbeya \\
\hline
\end{tabular}

\section{WTP Survey}

In total, 1892 CSI clients were interviewed over a period of six weeks (February - March 2004) regarding their stated willingness to pay higher prices for the service they had come to the clinic to receive. These services included DMPA injections, IUD insertions, and various consultations including gynecology, prenatal and general family planning. Clients made the decision to participate within the context of a standard informed consent process administered by study staff. ${ }^{4}$ For the predictive validity objective, 414 DMPA clients were enrolled in the panel study. ${ }^{5}$ Selection criteria included a stated intention to continue using the method for at least six months (i.e., two more injections), and willingness to be contacted for a follow-up interview. ${ }^{6}$ New DMPA acceptors were excluded because lack of experience with the method was assumed to impair their ability to give informed answers about their willingness to pay higher prices or about continued use of the method.

Clients were interviewed using a WTP instrument that adhered in many respects to the "best practices" guidelines for contingent valuation studies. ${ }^{7}$ First, the interviewer read a

\footnotetext{
${ }^{3}$ WTP predictions of non-DMPA clients were not validated, but this information was collected in order to allow CSI management to gauge stated WTP for other services.

${ }^{4}$ The process was as follows: the interviewer introduced herself to the client and gave a brief introduction to the study. The client then was told that if she chose to participate, her identity and the information she provided would be kept confidential. She was reminded that her participation was completely voluntary, that she could change her mind at any time, that her decision would not affect her treatment at the clinic, and that there would be no compensation for participating in the study. Finally, the interviewer asked the client if she agreed to participate.

${ }^{5}$ One client was dropped from the analysis because she switched during the index visit from injectable to IUD.

${ }^{6}$ Client consent to be contacted for follow-up was obtained separately from consent to participate in the WTP survey.

${ }^{7}$ Best practices recommendations for WTP surveys were developed by the National Oceanic and Atmospheric Administration (NOAA) in 1994. These include the following: (1) rely on personal interviews; (2) ask respondents about a future hypothetical occurrence rather than a historical event; (3) use close-ended questions that elicit the respondents' willingness to pay a specified increment for a service that
} 
brief scenario statement that summarized the circumstances surrounding the purchasing decision. $^{8}$ This statement was developed using results from a qualitative study carried out with CSI clients (Brelsford, 2004), which found that respondents wanted to know the reasons for the price increase, and how the additional revenue would be used by CSI. Questions to elicit client WTP followed a "bidding game" framework, as depicted in Figure 1.

Figure 1: Sequence of WTP Questions

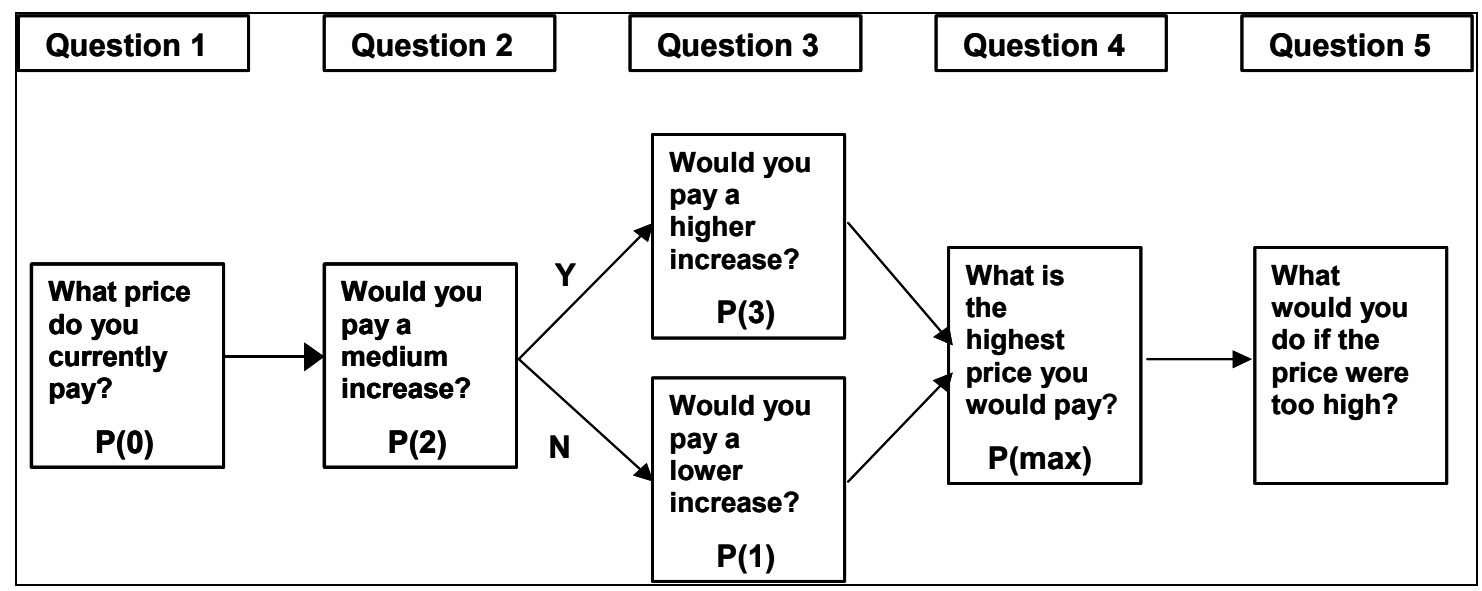

Each respondent first was reminded of the current price $(\mathrm{P}(0))$ for the service, and then was asked whether she would pay a price that was somewhat higher (which we denote as $\mathrm{P}(2)$ ). If she responded "yes" to $\mathrm{P}(2)$, then she was asked whether she would pay an even higher price $(\mathrm{P}(3))$. But if the response to $\mathrm{P}(2)$ was "no", she then was asked if she would pay a price between $\mathrm{P}(0)$ and $\mathrm{P}(2)$ which we denote as $\mathrm{P}(1)$. At the end of this process, all clients were asked to state the maximum price they would be willing to pay for the service, and what action they would take if the price increased to a level that was more than they were willing to pay or that they can afford. We make the assumption that clients who responded "yes" to either $\mathrm{P}(2)$ or $\mathrm{P}(3)$ would have also responded "yes" to $\mathrm{P}(1)$.

We also wanted to investigate whether client responses to WTP questions varied depending on client socioeconomic status. The survey instrument included questions on client and spouse educational attainment, earned income, and household characteristics

is familiar to them; (4) remind respondents that the price increment reduces other consumption; (5) include reminders that substitutes exist for the service in question; and, (6) question respondents about factors that might influence their preferences.

${ }^{8}$ The scenario statement used was the following: "The current price in Egyptian pounds for a DMPA injection is LE 3. This price includes the contraceptive product, the syringe and application of the injection by a nurse. In order to continue providing high-quality services and to ensure that we always have adequate stocks of family planning methods, CSI has decided to increase the price of the injection. I would like to ask you some questions about your response to possible changes in the price of this service.

Suppose that your income does not change but the price of this service goes up. Remember that you could get this same service at other places like the Ministry of Health or private physicians". 
including type of housing and ownership of various assets. Following a technique developed by Gwatkin, et. al. (2000) we used principal components analysis to compute an asset index from selected housing and asset variables. This index was used as a proxy for client ability-to-pay (ATP), under the assumption that an index constructed from several indicators better reflects ATP than does a single variable like self-reported income. Gwatkin's approach assigns an "asset score" to each client depending on the type and number of assets owned. These asset scores were used to divide the sample into ATP quintiles, which enabled us to search for associations between stated WTP of clients and their socioeconomic status as measured by the asset index.

\section{Price Increase}

For the predictive validity objective, a price increase for DMPA injections was implemented on 4 April, 2004, approximately one month following completion of the WTP survey. The price of a DMPA injection increased by LE 1 to 1.5 Egyptian pounds (depending on the current price in each clinic), or approximately 25 percent. The new price corresponded to the amount equal to $\mathrm{P}(1)$ in the baseline interview. Prices of other clinic services were not changed. Before a DMPA client left the study clinic, the attending nurse or counselor handed her a card that indicated the date of the next injection and the new price for the injection.

\section{Follow-up}

During a four-month observation period following the price increase, study staff monitored DMPA client follow-up patterns using existing CSI information systems. Interviewers from $\mathrm{CDC}$ contacted non-returning clients through a home interview to find out reasons why the client failed to return to the clinic, whether the price increase influenced their decision, whether they were still using a family planning method, and the current and future source of family planning services.

\section{Data Analysis}

For the first objective, we computed the proportion of clients that said they would accept the low, medium and high price increments by service and clinic. For the predictive validity objective, we constructed 2 by 2 tables in which the rows contained stated WTP (yes or no), and the columns contained actual client behavior (returned or did not return). An overall validity index consisting of the percentage of correct predictions was calculated. In addition, we computed the positive predictive validity and negative predictive validity of the $\mathrm{CV}$ method and corresponding 95 percent confidence intervals. For these data, the positive (negative) predictive value represents the conditional probability that any client would (would not) return for services given that they said that they would (would not) return. We also used McNemar's test (see Stokes et al,1995) to test for significant agreement between predicted and actual WTP. 


\section{Results}

\section{Stated WTP for CSI Services}

Figure 1 displays the results of analysis of client-stated WTP for other RH services. More than 80 percent of these clients said that they would pay higher prices, and more than half of all clients said that they would pay the highest of the three increments tested (information on magnitude of increments can be found in the notes to Figure 1). For the first three services listed in the figure, current prices and queried increments were similar across all clinics, and the proportions of clients verbally accepting these increments also showed little variation. Willingness to pay for IUD insertions was lower for all increments, probably because of the higher current price (median= LE 15) and larger absolute increments (LE 4.0, 7.5 and 12.5) for IUD insertions.

\section{Figure 1: Stated Willingness to Pay for CSI services}

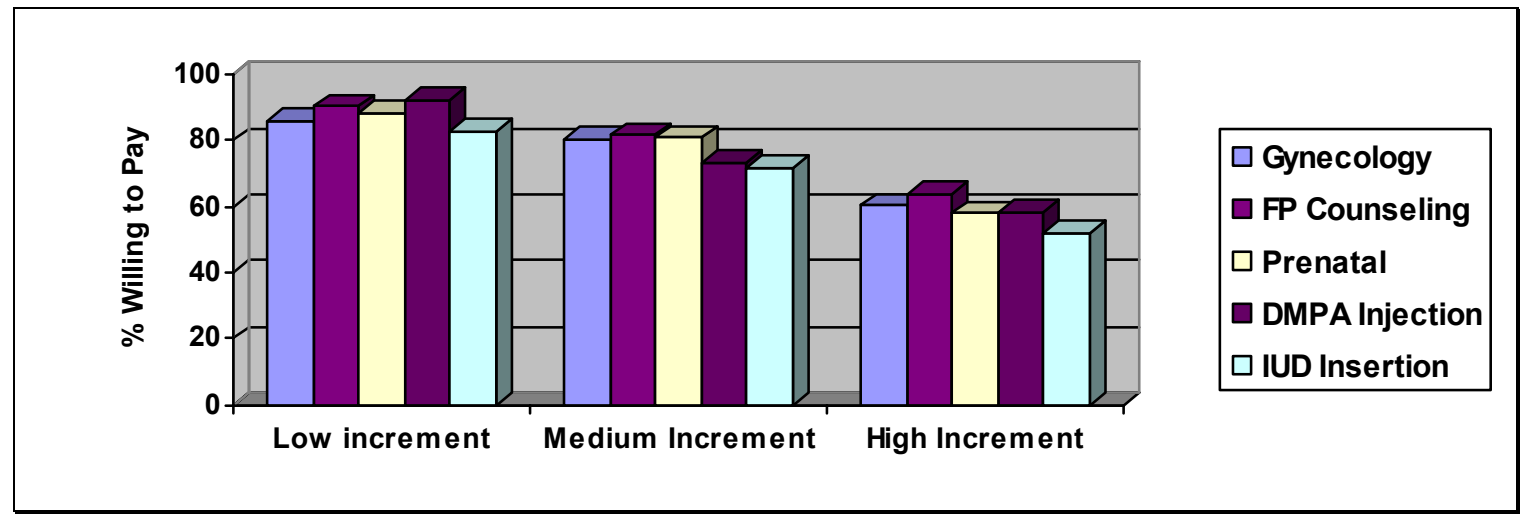

Note: The exchange rate in 2004 was approximately LE 6.2 to US\$1. For gynecology, family planning and prenatal consultations, the median current price was LE 5, and the medians of the low, medium and high increments were LE 1.5, LE 2.4 and LE 4.5, respectively. For DMPA injections, the median current price was $L E 3.5$, and the medians of the low, medium and high increments were $L E$ 1, $L E 2$ and $L E$ 3, respectively. For IUD insertions, the median current price was $L E 15$, and the medians of the low, medium and high increments were $L E 4, L E 7.5$ and $L E$ 12.5 , respectively.

\section{Predicted Changes in Client Use of Services Following a Price Increase}

A concern among program managers is that price increases will encourage clients to abandon the use of reproductive health services. We found very consistent responses across all CSI services to the question, "If the price of this service were to increase to a level that you did not want to pay or could not afford, where would you go for this service?" For all services, approximately one-third of clients said that they would continue using the service at CSI regardless of the magnitude of the price (data not shown). Another third said that they would go to lower-priced MOHP outlets for services. The remaining third of clients included those who said they would switch to a lower-priced method (in the case of FP), would go to a commercial sector outlet, or did not know where they would go. Only 2 percent of DMPA clients and 1 percent of IUD 
clients said that the price increases would result in discontinuation of family planning altogether.

\section{Predicted Impact of Price Changes on CSI Utilization and Revenues}

If client predictions about future purchasing behavior are accurate, then results of the WTP survey can be used to predict utilization and revenues in CSI clinics after a price increase. Figure 2 presents an example of such an analysis using data for DMPA injections. The bars represent the proportion of all clients willing to pay each specific price, and the line represents total revenue that would be earned at each price level, assuming that client predictions are valid. The current price is LE 3.5 , and 100 percent of clients are assumed to be willing to pay this price since it is the current price. WTP declines as the price increases; thus, when the price reaches LE 6.5 , slightly more than half of the clients say they are willing to pay.

\section{Figure 2: Predicted changes in Utilization and Revenues related to CSI Provision of DMPA Injections, by Price $(n=414)$}

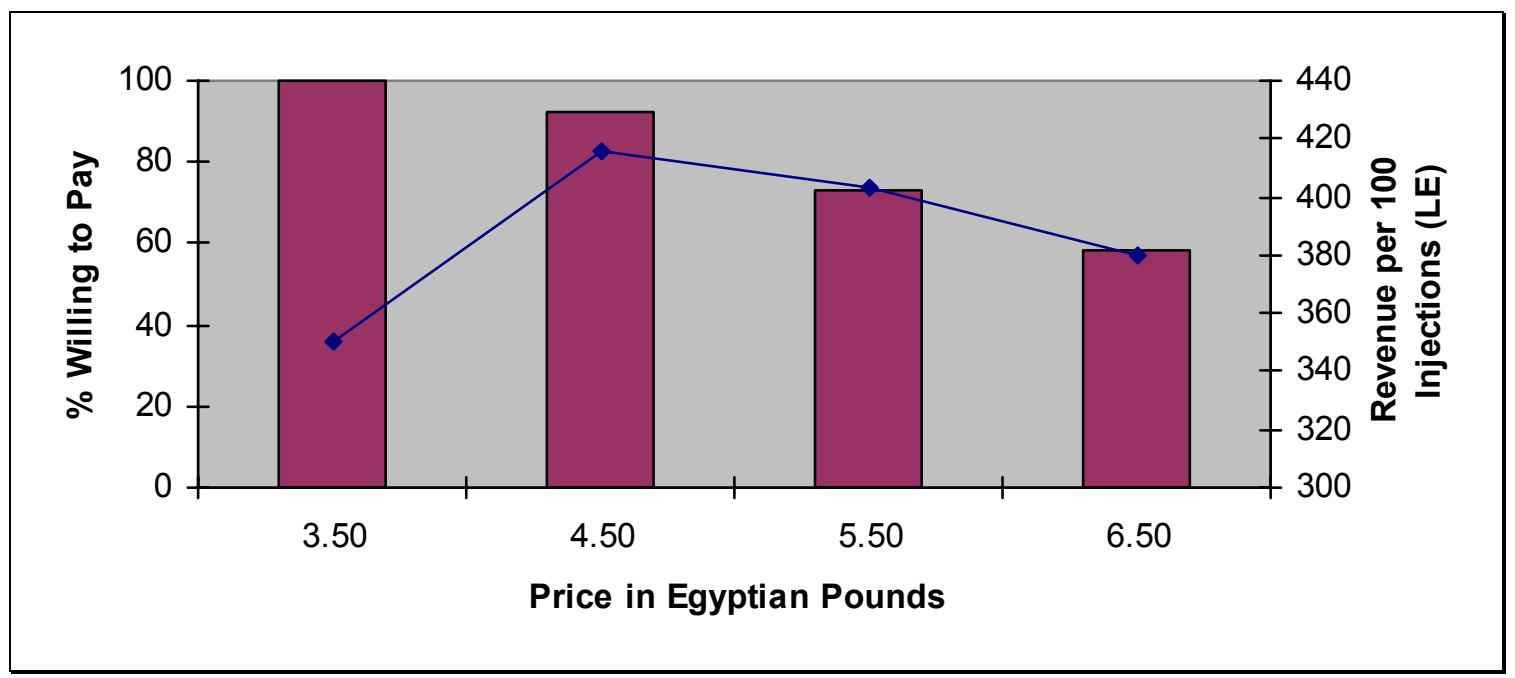

The revenue impact of these changes in price and utilization is indicated by the line, and the appropriate scale is found on the right-hand vertical axis. At the current price of LE 3.50 and 100 percent willingness to pay, total revenue from 100 clients would be LE 350 . If the price were to increase to LE 4.50, total revenue would rise to LE 416, even though utilization would decline by $7-8$ clients. Increasing the price to LE 5.50 would result in lower total revenue than at the price of 4.50 , and a total of 27 lost clients. A further increase in price to LE 6.50 would result in still lower total revenue and loss of 42 clients. Thus, raising the price for DMPA injections above LE 4.50 would make little sense, since both revenue and utilization would decline. Similar analyses for other CSI services can be found in Appendix 1.

A natural extension of this analysis is to examine what would happen to the overall clinic-level cost recovery ratio if price increases in the survey had been implemented (see table 1). The cost recovery ratio is computed by dividing total clinic income by total 
clinic expenditures. A value greater than 1.0 indicates that clinic income covers more than 100 percent of clinic costs, while a value less than 1.0 indicates that clinic income does not fully cover costs. During the six-month period January - June 2004, cumulative cost recovery ratios in the six CSI study clinics ranged from 0.79 to 1.02 , with an average of 0.91. If CSI had implemented the low price increase for all five services, and client demand changed as predicted in the CV survey, cost recovery ratios in all clinics would have increased (see third column). The predicted impact on utilization would be a reduction of 2.6 to 8.2 consultations per day, depending on the clinic. If the medium price increase had been implemented, cost recovery ratios would have increased further in four of the six clinics. But declines in utilization are much steeper with the medium price increase, ranging from 5.2 to 16.2 fewer consultations per day depending on the clinic.

Table 1: Predicted Impact of Price Changes on Cost Recovery Ratio (CRR) and Clinic Utilization, by Clinic

\begin{tabular}{|c|c|c|c|c|c|}
\hline Clinic & $\begin{array}{l}\text { CRR Jan- } \\
\text { June } 2004\end{array}$ & $\begin{array}{l}\text { CRR - if } \\
\text { Low } \\
\text { Increment } \\
\text { had been } \\
\text { Implemented }\end{array}$ & $\begin{array}{l}\text { CRR - if } \\
\text { Medium } \\
\text { Increment } \\
\text { had been } \\
\text { Implemented }\end{array}$ & $\begin{array}{l}\text { Change in } \\
\text { utilization } \\
\text { (visits per } \\
\text { day) low } \\
\text { increment }\end{array}$ & $\begin{array}{l}\text { Change in } \\
\text { utilization } \\
\text { (visits per } \\
\text { day) } \\
\text { medium } \\
\text { increment }\end{array}$ \\
\hline Delengat & 0.97 & 1.04 & 1.07 & -3.4 & -6.1 \\
\hline Hosh Issa & 0.88 & 0.91 & 0.86 & -8.2 & -16.2 \\
\hline Zefta & 1.02 & 1.08 & 1.05 & -3.4 & -7.3 \\
\hline Zakazik & 0.97 & 1.01 & 1.06 & -2.7 & -5.2 \\
\hline Banha & 0.87 & 0.90 & 0.94 & -6.3 & -8.6 \\
\hline Ayat & 0.79 & 0.89 & 0.93 & -2.6 & -6.1 \\
\hline Total & 0.91 & 0.96 & 0.98 & -26.7 & -49.4 \\
\hline
\end{tabular}

Note: for the low increment, absolute changes in clinic revenues over the six-month period would range from LE 2,070 to LE 5,648 (US\$334-US\$911). For the medium increment, absolute changes in clinic revenues would range from a loss of LE 1252 to a gain of LE 7823 (loss of US\$202 to gain of US\$1262).

4. Relationship between Client Ability to Pay (ATP) and Willingness to Pay

A further question is whether relatively poorer CSI clients would be disproportionately affected by an increase in prices. Figure 3 presents information on the relationship between client ability to pay (as measured by the household asset index) and client willingness to pay the three prompted price increments. The CSI client population has been divided into five equal groups arranged according to household asset scores; for 
example, the "lowest" group is the bottom 20 percent of the distribution of asset scores, and the "highest" group is the top 20 percent of the distribution. If a strong relationship existed between ATP and stated WTP, we would expect to see the lines rising sharply from left to right, indicating that relatively wealthier clients were more willing to pay the prompted increments. For all three increments an upward trend is visible, and is statistically significant at the .05 level (chi-square test for trend). But most of the upward movement occurs in the highest quintile; the trend-line in the lower four quintiles is nearly flat, and is not statistically significant for any of the three increments. Thus, it appears that stated WTP does not vary much among CSI clients, especially in the lower 80 percent of the asset index distribution.

\section{Figure 3: Stated WTP Different Price Increments, by Wealth Index Quintiles $(n=1892)$}

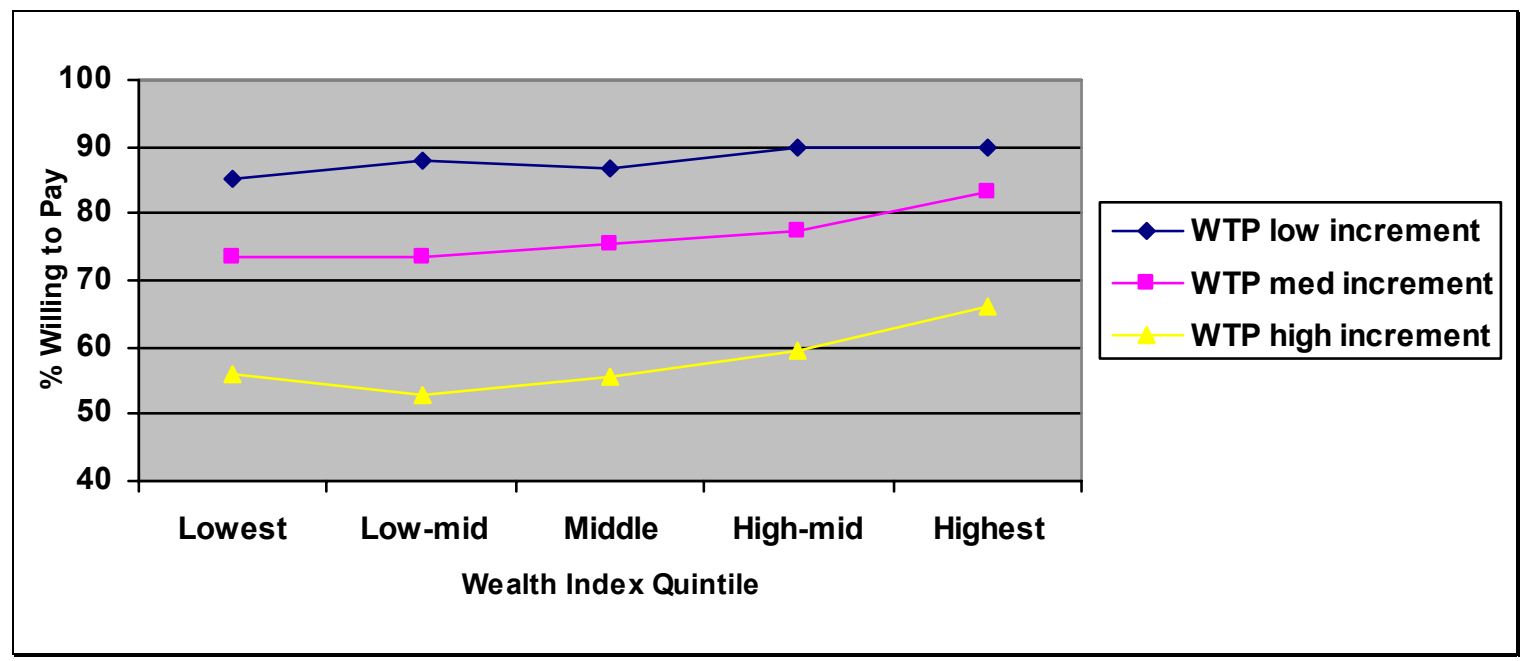

Notes: P-values for the chi-square test for trend including all five quintiles: low increment $\mathrm{p}<.043$; medium increment $\mathrm{p}<.0006$; high increment $\mathrm{p}<.0007$. P-values for the chi-square test for trend including lower four quintiles only: low increment $\mathrm{p}<.101$; medium increment $\mathrm{p}<.181$; high increment $\mathrm{p}<.253$.

\section{Predictive Validity of the WTP Survey}

Table 2 presents findings on predictive validity of the WTP survey for all DMPA users. In the aggregate, the survey predicted that 383 clients out of 414 (92.5\%) would return for a subsequent DMPA injection, and that 31 (7.5\%) would not return. Actually, 315 clients of the 414 (76.1\%) returned, while 99 clients (23.9\%) did not. Estimated positive predictive validity (i.e. those who actually returned given that they had said they would return) was $76.8 \%$, and estimated negative predictive validity (those who did not return given that they had said they would not return) was $32.3 \%$, which indicates that the WTP survey was much more effective in predicting who would return than who would not return. The $\mathrm{p}$-value for McNemar's test was $<0.0001$, suggesting that the proportion 
who said they would return $(92.5 \%)$ was significantly different than the proportion who actually returned (76.1\%), accounting for the pairing of the data.

Table 2: Comparison of Client Stated Willingness-to-Pay and Actual Behavior in Six CSI Clinics (percent of total in parentheses)

\begin{tabular}{|l|c|c|c|}
\hline \multirow{2}{*}{$\begin{array}{l}\text { Client Said She Would Return } \\
\text { to Clinic After Price Increase }\end{array}$} & \multicolumn{2}{|c|}{$\begin{array}{c}\text { Client Returned to Clinic } \\
\text { After Prices Increased }\end{array}$} & \multirow{2}{*}{ Total } \\
\cline { 2 - 4 } & Yes & No & \\
\hline Yes & 294 & 89 & 383 \\
& $(71.0 \%)$ & $(21.5 \%)$ & $(92.5 \%)$ \\
\hline No & 21 & 10 & 31 \\
& $(5.1 \%)$ & $(2.4 \%)$ & $(7.5 \%)$ \\
\hline Total & 315 & 99 & 414 \\
& $(76.1 \%)$ & $(23.9 \%)$ & $(100 \%)$ \\
\hline
\end{tabular}

Table 2 also shows the proportion of clients who made correct versus incorrect predictions. Clients in the group making correct predictions - defined as those who said they would return and did return, or those who said they would not return and did not return - totaled 304 clients $(294+10)$, or 73.4 percent of all clients. The remainder of clients made incorrect predictions (26.4\%); most were clients who predicted that they would return but did not. These clients are the most troubling from the perspective of a program manager using WTP to forecast revenue and utilization. Expected revenue from these clients would not materialize, and utilization would be much lower than projected.

Table 3 summarizes the findings of the follow-up survey that was conducted in order to determine client reasons for not returning to CSI for subsequent DMPA injections. CDC interviewers succeeded in locating and interviewing 97 of the 99 non-returning clients. Of these 97 clients, approximately half were still using a method of family planning, and half were not. The most common reason for not continuing to use DMPA at CSI was the experience of side-effects related to the method, accounting for half of non-returns overall. It is well-known that many users of injectable contraceptives experience side effects - including changes in menstrual bleeding patterns, weight gain and headaches that result in high levels of discontinuation in the first year of use (WHO, 1983; Hall et. al, 1994). CSI's own service statistics from the same time period during the previous year showed a discontinuation rate for DMPA clients of approximately 25 percent, nearly identical to the rate of 23.9 percent observed in our study. 
Table 3: Reasons for Client Discontinuation of DMPA use at CSI

\begin{tabular}{|l|c|c|c|}
\hline \multirow{2}{*}{$\begin{array}{l}\text { Main Reason for Not Returning } \\
\text { for Next DMPA Injection }\end{array}$} & \multicolumn{2}{|c|}{ Current Use of Family Planning } & \multirow{2}{*}{} \\
\cline { 2 - 3 } & Still Using FP & Not Using FP & Total \\
\hline & & & \\
\hline Method-related problems & 29 & 20 & 49 \\
\hline Access Problems & 8 & 6 & 14 \\
\hline No current need for FP & 0 & 17 & 17 \\
\hline Economic problems & 9 & 2 & 6 \\
\hline Others & 3 & 3 & $\mathbf{9 7}$ \\
\hline & & & \\
\hline Total & 49 & 48 & \\
\hline
\end{tabular}

Among clients still using a FP method, economic problems - including difficulty affording the higher price, or more generally, a problem with lack of resources to pay for FP - was the second-most common reason for leaving CSI. Six of the 9 persons in this category reported having gone to the MOHP for subsequent service, while three obtained their method from private physicians or a pharmacy (data not shown). Meanwhile, only two clients in the non-use group cited economic problems as the main reason for not returning to CSI. Other important reasons related to problems with access to CSI clinics and, in the non-use group, a lack of a current need for FP (e.g. divorce, husband's travel, desire for more children).

Thus, while the baseline survey encouraged clients to relate their continuation intentions exclusively to hypothetical changes in price, the follow-up survey showed that factors other than the price increase motivated nearly 90 percent of the decisions to discontinue use of DMPA at CSI clinics. In other words, the true relationship between client WTP and subsequent purchasing behavior is distorted by non-economic reasons that were unforeseen by clients at the time of the baseline interview. Table 4 presents the results of an analysis of the relationship between stated and observed WTP in which clients discontinuing for non-economic reasons are removed.

Table 4: Comparison of Client-Stated Willingness-to-Pay and Actual Behavior (After Removing Clients who Discontinued for Non-economic Reasons)

\begin{tabular}{|l|c|c|c|}
\hline \multirow{2}{*}{$\begin{array}{l}\text { Client Said She Would Return } \\
\text { to Clinic After Price Increase }\end{array}$} & \multicolumn{2}{|c|}{\begin{tabular}{c} 
Client Returned to Clinic \\
\multirow{2}{*}{ After Prices Increased }
\end{tabular}} \\
\cline { 2 - 3 } & Yes & No & Total \\
\hline Yes & 294 & 11 & 305 \\
& $(89.6 \%)$ & $(3.4 \%)$ & $(93.0 \%)$ \\
\hline No & 21 & 2 & 23 \\
& $(6.4 \%)$ & $(0.6 \%)$ & $(7.0 \%)$ \\
\hline Total & 315 & 13 & 328 \\
& $(96.0 \%)$ & $(4.0 \%)$ & $(100 \%)$ \\
\hline
\end{tabular}


In the aggregate, predictive validity of the CV survey is much improved after removing clients who discontinued for non-economic reasons. The survey predicted that 305 clients out of $328(93 \%)$ would return, and 315 clients $(96 \%)$ did, in fact, return. At the individual level, the proportion of clients making correct predictions increased from 73.4 percent to 90.2 percent, an increase of nearly 17 percentage points. Of the 32 incorrect predictions, 11 were clients who said they would come back but did not; these were more than offset however by the 21 clients who said they would not return, but did. Positive predictive validity increased to 96.4 percent $(95 \%$ CI 93.8\%, 98.2\%) but negative predictive validity declined to 8.7 percent $(95 \%$ CI $1.6 \%, 26.8 \%)$. The p-value for McNemar's test was 0.11 , indicating that the proportion who said they would return $(93 \%)$ is not significantly different than the proportion who actually returned $(96 \%)$, when the data are paired.

\section{Conclusions}

Stated WTP for all services was high in the six CSI clinics, but the proportion of clients WTP higher prices declined as the magnitude of the queried increments increased. WTP did not exhibit much variation by client socio-economic status, particularly for clients in the lower 80 percent of the distribution of asset index values. This is unexpected, since theory predicts that WTP should vary directly with client ability to pay. There are several possible explanations for this finding: the asset index could be an invalid measure of ATP, the spectrum of ATP of CSI clients may be narrow, or customer loyalty to CSI may be extraordinarily high. A likely explanation is that the queried increments were so low relative to client financial resources that there was little doubt that clients would be WTP for services that they value. In any case, the findings suggest that somewhat higher fees would be acceptable to CSI clients across the SES continuum. ${ }^{9}$

Predicted changes in revenue and utilization from the low and medium increments would increase cost recovery in almost all CSI clinics, but as predicted by economic theory, these changes also would result in fewer visits to clinics. CSI managers must weigh the importance of additional revenue against the reduced number of visits when deciding whether and how much to increase prices. A benefit of using the WTP technique is that these decisions can be made on the basis of valid and reliable evidence. In the CSI study clinics, concern about the impact of price increases on overall FP utilization should be lessened by the evidence that almost no clients would discontinue FP use altogether. Rather, clients would continue to obtain FP, but from different sources.

This is the second recent study to evaluate predictive validity of a WTP survey at the individual client level. ${ }^{10}$ Although programs are interested mainly in validity of WTP in the aggregate, individual-level studies provide the strongest evidence for validity, and discontinuers can be followed up to understand the reasons for leaving the program. We

\footnotetext{
${ }^{9} \mathrm{We}$ also asked clients their opinion of the current CSI prices, and more than 85 percent said they thought CSI prices were "inexpensive" or "reasonable".

${ }^{10}$ The main differences between the current study in Egypt and a previous study in El Salvador were: (1) our focus on users of a longer-duration injectable (DMPA), and (2) our use of a more detailed scenario statement.
} 
found that predictive validity was low when all reasons for client discontinuation were included; although nearly three-quarters of DMPA clients correctly predicted their future purchasing behavior, more than 20 percent said they would return for a follow-up injection but did not return. At the aggregate level, the WTP survey under-predicted drop-outs by 17 percentage points. But when we removed clients from the analysis who discontinued for reasons other than the price increase, the proportion of clients making correct predictions increased to over 90 percent, and the aggregate-level prediction was almost identical to the proportion of clients who returned. This level of predictive validity should be satisfactory for program managers wishing to use WTP surveys to predict WTP for DMPA clients. But utilization predictions for methods like DMPA (which have high discontinuation for method-related reasons) must be adjusted downward using data on method discontinuation from program statistics or from the literature. 


\section{Appendix 1: Predicted Changes in Revenue and Utilization for Other CSI Services}

These figures provide information on changes in revenue and utilization that could be expected if CSI changed its prices and the predictive validity of the WTP survey was high for these services. In each figure the bars show the percentage of clients accepting each price increase, and the line depicts the trend in revenues associated with different prices and utilization levels.

1. IUD Insertions

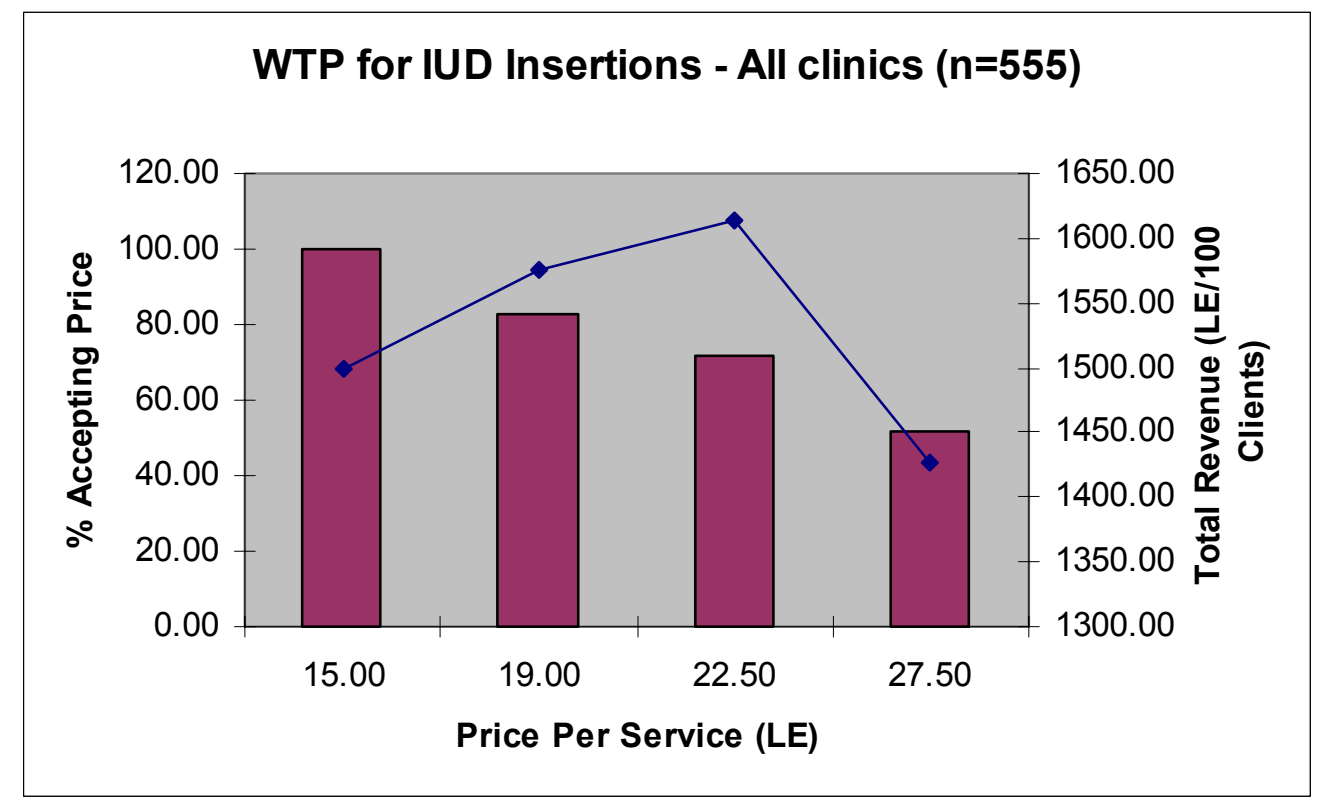

2. Family Planning Consultations

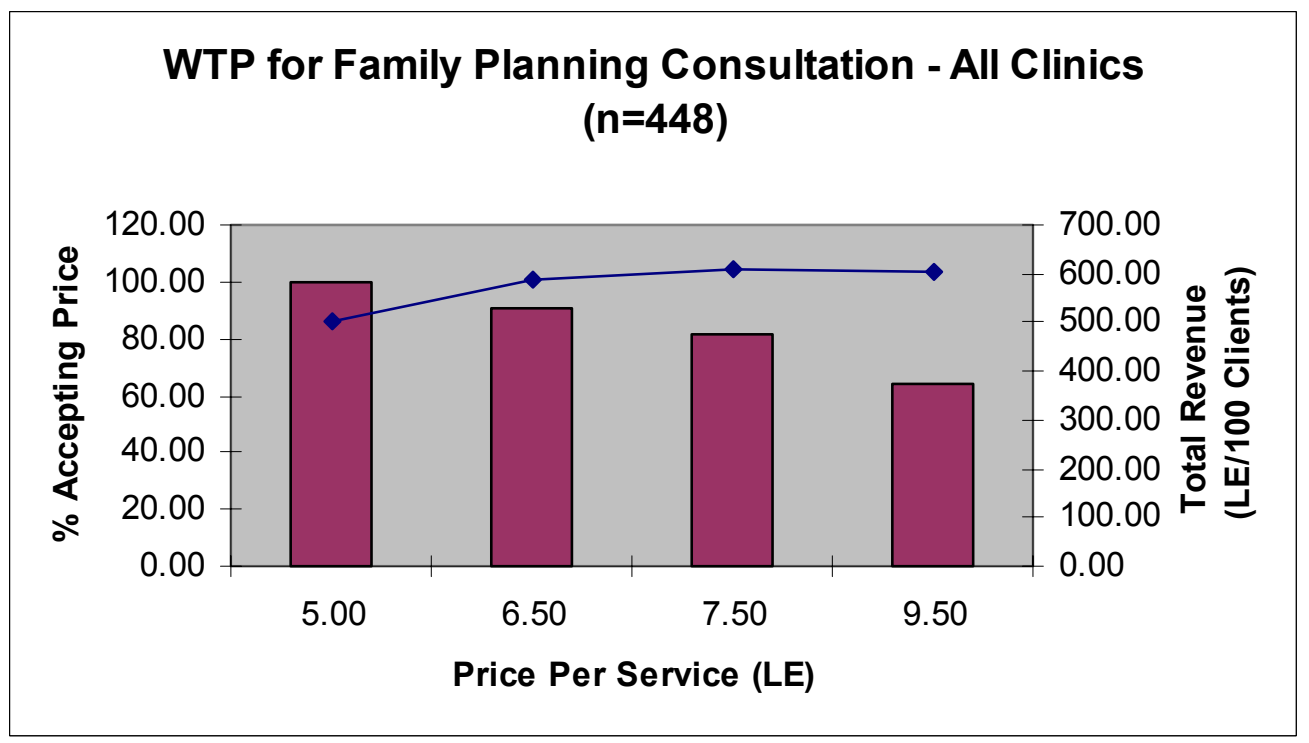


3. Gynecology

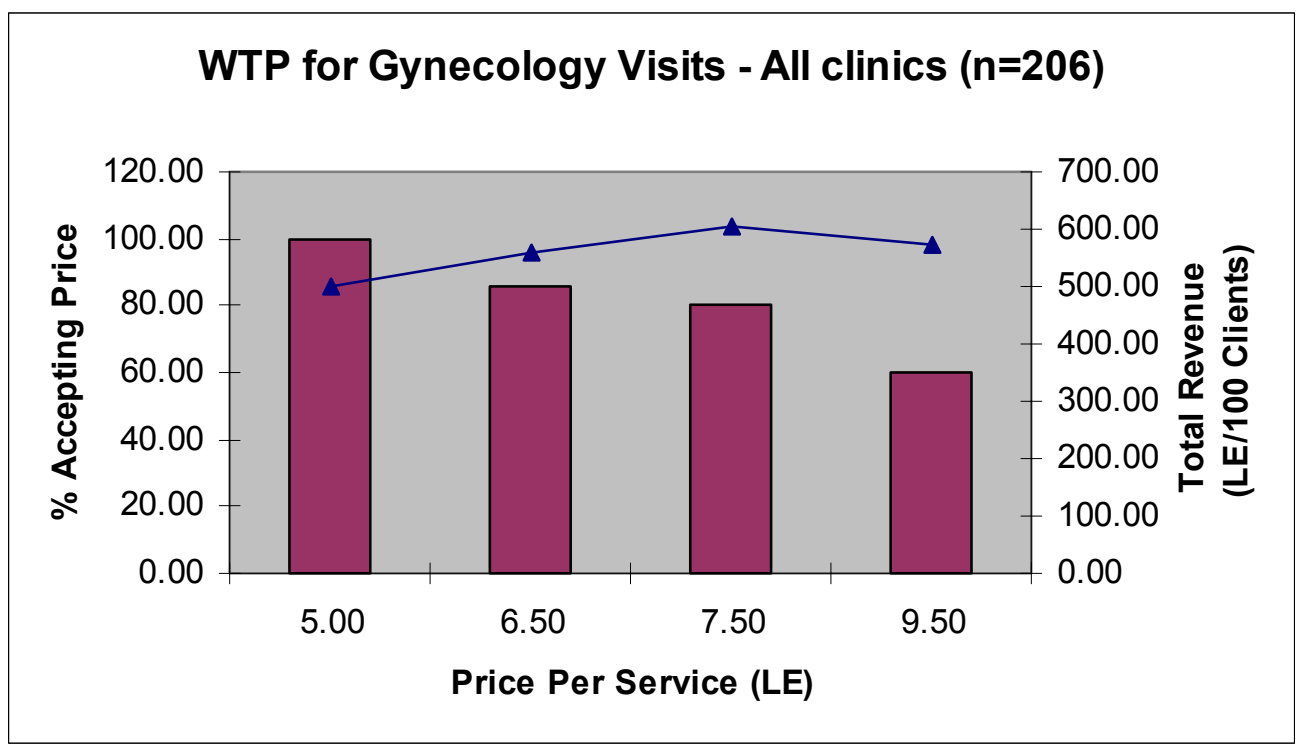

4. Antenatal Care

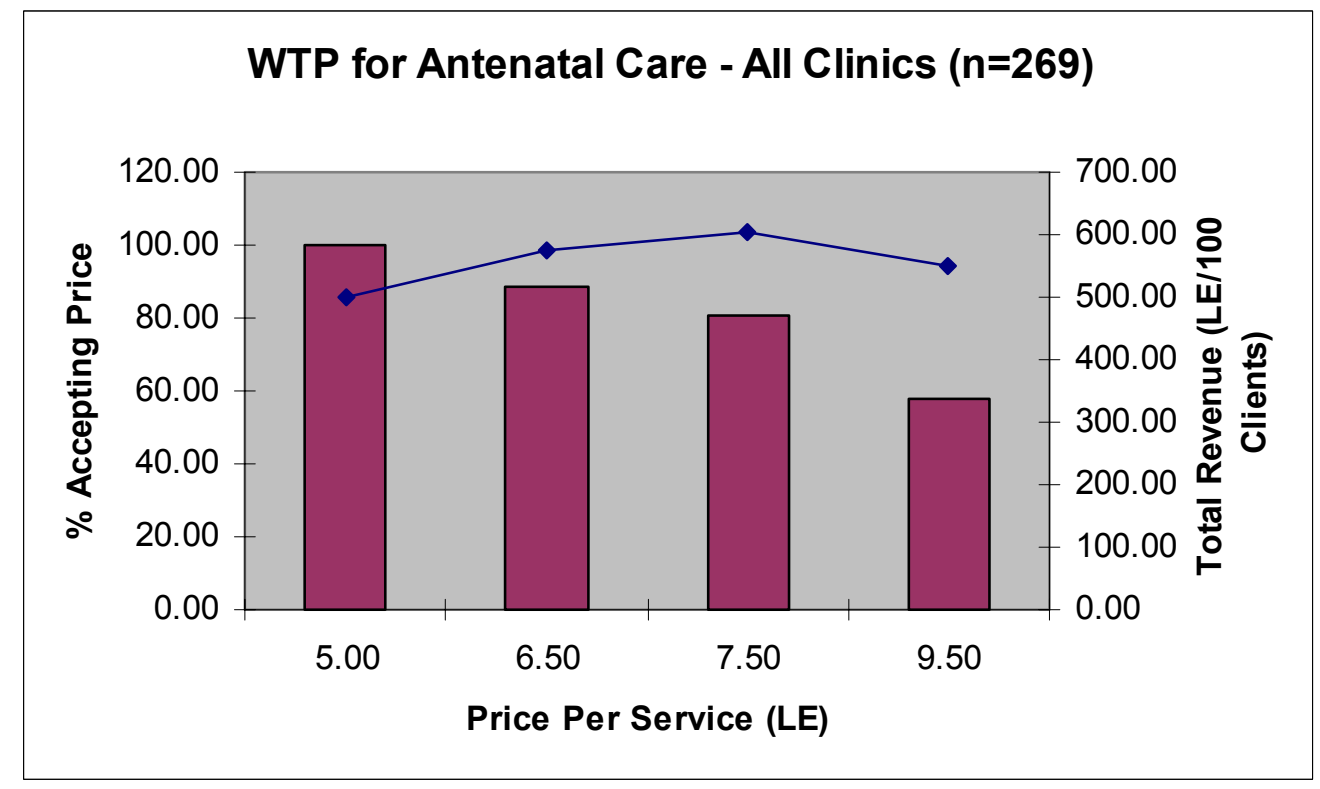




\section{References}

Bratt JH, Weaver MA and Araya, JD (2004). Criterion Validity of Willingness to Pay for Increased Prices of Contraceptive Injections in El Salvador. Submitted to Health Policy and Planning July 2004.

Brelsford E (2004). Validation of a Survey-based Approach for Predicting Willingness to Pay for Reproductive Health Services: Instrument Validation Qualitative Research in Cairo, Egypt. Family Health International, unpublished report, June 24, 2004.

Cairo Demographic Center (1997). Clients Payments and Willingness to Pay for Family Planning in Egypt. Cairo: National Population Council, Institutional Development Project.

El-Zanaty F and Way A (2004). Egypt Interim Demographic and Health Survey, 2003. Cairo: Ministry of Health and Population and National Population Council, Calverton, MD: ORC Macro.

Foreit JF and Foreit KGF (2003). The Reliability and Validity of Willingness to Pay Surveys for Reproductive Health Pricing Decisions in Developing Countries. Health Policy 63 (2003) 37-47.

Griffin CG, J Briscoe, B. Singh, R Ramasubban and R Bhatia (1995). Contingent Valuation and Actual Behavior: Predicting Connections to New Water Systems in the State of Kerala, India. The World Bank Economic Review Vol. 9 No.3 373 - 395.

Hall, PE and World Health Organization Task Force on Research on Introduction and Transfer of Technologies for Fertility Regulation (1994). The introduction of Cyclofem into national family planning programmes: Experience from studies in Indonesia, Jamaica, Mexico, Thailand and Tunisia. Contraception 49(5): 489-507.

Onwujekwe O (2004). Criterion and Content Validity of a Novel Structured Haggling Contingent Valuation Question Format versus the Bidding Game and Binary with Follow-up Format. Social Science and Medicine 58:525-537.

Stokes ME, Davis CS, and Koch GG (1995). Categorical Data Analysis Using the SAS® System. SAS Institute: Cary, NC.

World Health Organization (1983). Multinational Comparative Clinical Trial of Longacting Injectable Contraceptives: Norethisterone Enanthate given in Two Dosage Regimens and Depot-medroxyprogesterone Acetate: Final Report. Contraception 28(1): $1-20$. 